\title{
Mechanical simulation design of the shaft type hybrid mechanical arm based on Solidworks
}

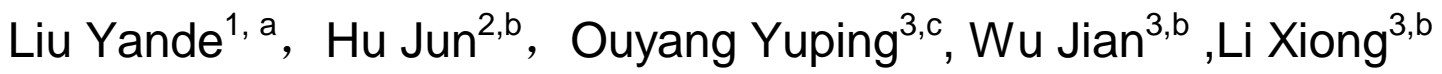 \\ ${ }^{1}$ School of Mechanical and Electronical Engineering, East China Jiaotong University,Nanchang \\ Jiangxi 330013,China \\ a1258622092@qq.com, b1825868002@qq.com, '704733641@qq.com
}

Keywords: Mechanical arm; SolidWorks; Simulation; Finite element.

Abstract. Industrial robot arms are widely used in industry, The analysis of the force situation has important significance. In this paper, a kind of four degree of freedom of the combination of the mechanical arm has designed by using a three-dimensional software SolidWorks, which is used for conveying materials to stamping equipment. Based on the principle of 3D simulation design, this paper designed dynamically the each parts of the robot arm, including the structure of base, big arm, small arm, clamping manipulator. at last, all the parts are assembled. And then selected the appropriate materials for structural calculation, process analysis, strength check on the important parts by using finite element analysis software, And then according to the results of the check to modify the original parts. Finally, Completing the design by selecting the appropriate driving mode and transmission scheme.

\section{Introduction}

Industrial robot arms are widely used in industry, The analysis of the force situation has important significance. The motion plan and design of key parts, the working process of the strength and service life are directly related to production safety. Based on the principle of 3D simulation design, this paper designed dynamically the each parts of the robot arm, including the structure of base, big arm, small arm, clamping manipulator. at last, all the parts are assembled. And then selected the appropriate materials for structural calculation, process analysis, strength check on the important parts by using finite element analysis software, And then according to the results of the check to modify the original parts. Finally, Completing the design by selecting the appropriate driving mode and transmission scheme.

\section{Three-dimensional solid modeling}

\section{Base and turntable design}

\section{Base design}

The base is the support of the whole robot body. In order to ensure the stability of the manipulator, the solid cast iron is used as the support. The Base structure design is shown in picture 1, the base is fastened by double nut and designed the reinforced bar in the turntable to increase its stiffness. The back end of the base is a junction box, all motor drive signals and feedback signals are in and out.

\section{Turntable design}

The shape of the turntable is shown in Fig.2, Turntable is the waist of the mechanical arm, Its importance is self-evident. The internal servo motor and the rotary disc are driven by a gear belt, that can protect the motor at the same time to achieve the motor at the same time stepper motor stepper precise control. A single acting hydraulic cylinder is installed at the back of the turntable, When the load is too large or the required control precision is not high, the hydraulic cylinder can be used as the power source. 


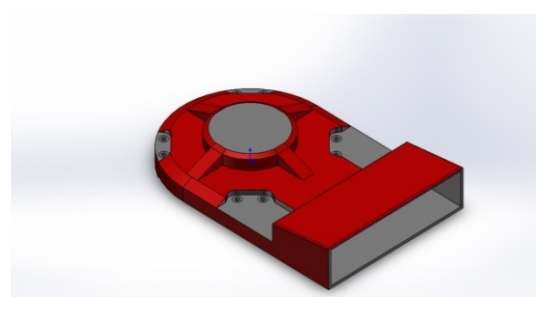

Fig.1 The Base structure

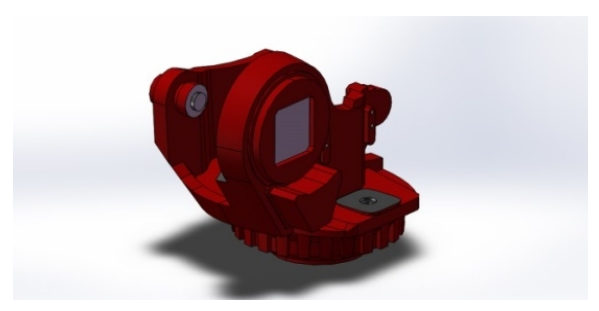

Fig.2 The Base turntable

\section{Arm design}

\section{Big arm design}

The arm is equal to the elbow of a man's arm, what the arm design should pay attention to is considering how to reduce material consumption and increase the balance of the structure on the premise of meeting the requirements of the basic dimensions. In this design, The material of arm adopted 1060 alloy, which has little quality and strength meets the design requirements also. In addition, For strengthening the structural and balanced consideration. The big arm is designed as a " $\mathrm{S}$ " shape for its axis is coincident with the axis of the small arms, which greatly improve the structural balance. The big arm design is shown in Fig.3.

\section{Small arm design}

The material consumption of Small arm is less than the big arm, the volume is also light . In order to adapt to the condition of grabbing a large range of work piece, To separate the front of the small arms and connected with the small arm mandrel. At the same time, adding a telescopic hydraulic cylinder, making the robot more adaptable. That is a innovation point on small arm design. The small arm design is shown in Fig.4.

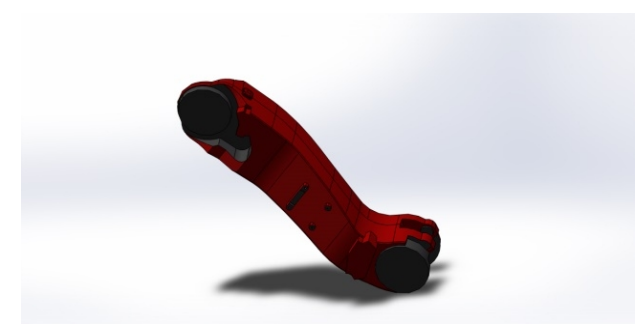

Fig.3 Big arm

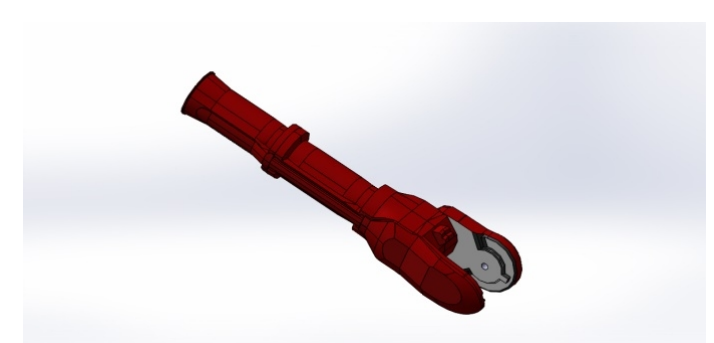

Fig.4 The small arm

\section{Clamping manipulator Design}

Device for clamping material of mechanical arm is also called end effector, It is the imitation of the human finger joints grab and hold (adsorption) parts of the material, which has features of small size and high precision. The mechanical arm designed by this design is used to clamp the thin wall stamping parts. In the mechanical hand clamping work piece movement, if the clamping force is not enough to clamp the work piece, if the clamping force is too large, it will damage the work piece. The mechanical arm end effector can be divided into: clamp type and adsorption type, special operation type, bionic multi finger. As the clamping manipulator in this design is designed to transport material punching, and it does not need to bionics fingers, so only need to design the clamp which can grasp the workpiece from the different angle. The design adopts two clamping jaws, and the inner side of the clamping jaw comprises a tooth type non slip groove which is shown in Fig.5. The releasing and clamping of the workpiece is achieved through the opening and closing of the two clamping jaws.

According to the movement characteristics of finger opening and closing into rotary and transitional. This design adopted lever type transmission mechanism. The Clamping manipulator part is shown in Fig.6. 


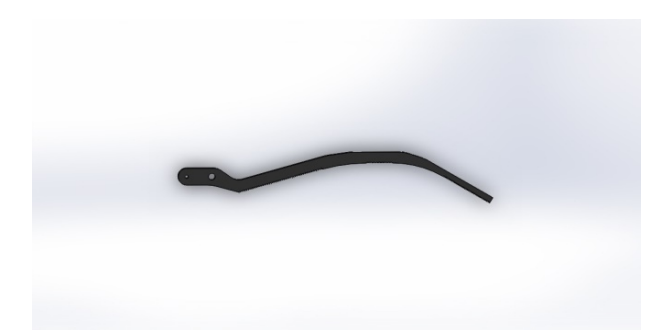

Fig.5 Mechanical finger shape

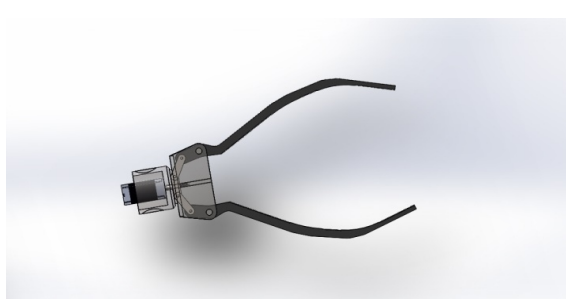

Fig.6 Clamping manipulator part

Linear motor of Clamping manipulator makes the two lever mechanism in series for achieving the motion of fingers. In order to make sure the smooth closure of fingers and the closure does not rebound, That need to make the linear motor lead screw with self locking which can lock clamping force.

\section{Arm assembly}

\section{Base assembly}

The assembly process of the base is simulated under the assembly interface of solidworks software. First, the base is fixed on the machine frame through bolts. Then, the turntable and the base are installed together. The fixed gear is mounted on the base, which is connected with the servo motor through a gear belt. Then the base can achieve the action of "turn waist". Finally, the hydraulic cylinder and the shoulder joint servo motor can be installed. The Base assembly drawing as shown in Fig.7.

\section{Arm assembly}

Arm assembly is the process of assembling a large arm and a small arm through an arm connector. Two servo motors are needed to realize the movement of the elbow joint and the telescopic function of the arm. First, the large arm fixed, the connector and the big arm turntable installed together. The servo motor is mounted on the turntable, which can complete the movement of elbow joint. In the end, the small arms are mounted to the connector. The Arm assembly drawing as shown in Fig.8.

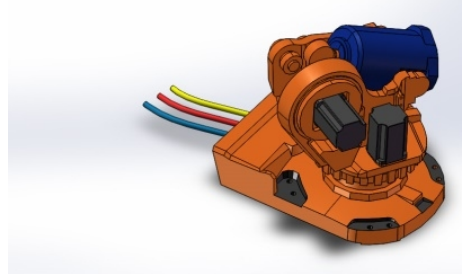

Fig.7 Base assembly drawing

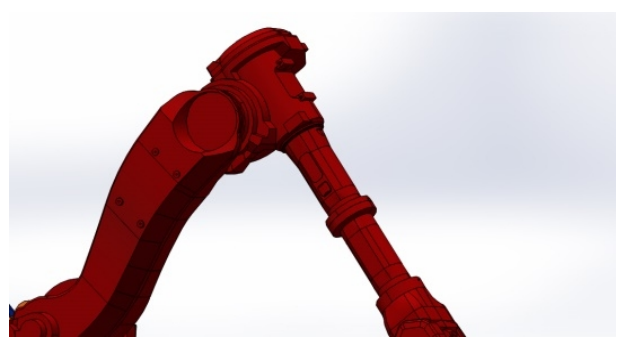

Fig.8 Arm assembly drawing

\section{Clamping manipulator assembly}

First, the clamping jaw frame is fixed and the gripper and gripper pins fitted to the rack. The other end of the clamping jaw is connected with the small connecting rod together and arranged on a square nut of the linear motor. Then, the assembly is completed and implement the clamping jaw movement. The gripper assembly together with the servo motor on the wrist of arm that can realize the connection of the clamping arm and the small arm and increase the degree of freedom of the clamping hand rotation.

\section{General assembly drawing}

The assembly of all parts can be assembled together to complete the assembly, as shown in Fig. 10.

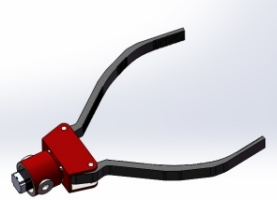

Fig.9 Clamping manipulator assembly drawing

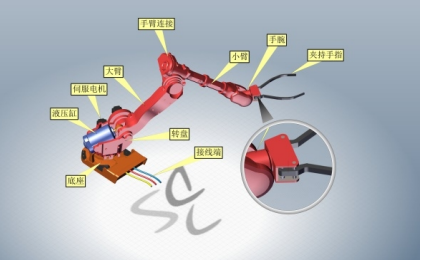

Fig. 10 General assembly drawing 


\section{Strength check of typical parts}

\section{Material selection}

According to the working condition of the arm to select the material of arm based on the principle of high efficiency and energy saving and reducing of the environmental pollution. Firstly, the base selection of gray cast iron can meet the requirements by comprehensively considering from the quality, stiffness, inertia force. Secondly, as the turntable is susceptible to fatigue, its material adopted 45 steel for quenching and tempering treatment. Thirdly, The arm is under load, which can not appear large strain and fracture, that is to say to the arm must have enough strength and can be well controlled. Its characteristics is small density, big strength and small moment of inertia. To sum up, the material of the arm should choose light alloy material.

\section{Analysis of natural vibration frequency of base}

All objects have their inherent vibration properties. The natural frequency of the turntable should be analysed, in order to avoid the same resonance which could cause the loss of parts and other adverse consequences when the natural vibration frequency of the turntable is the same as the frequency of power source load. The five sets of natural frequencies of the base as shown in Fig.11.

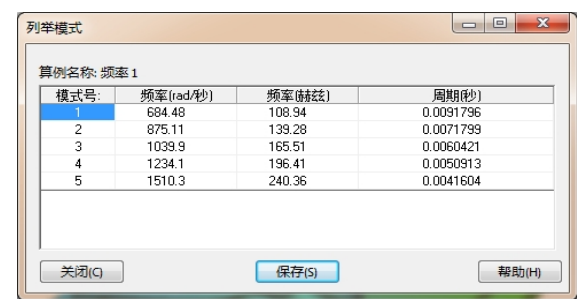

Fig.11 Table of natural frequency analysis of turntable

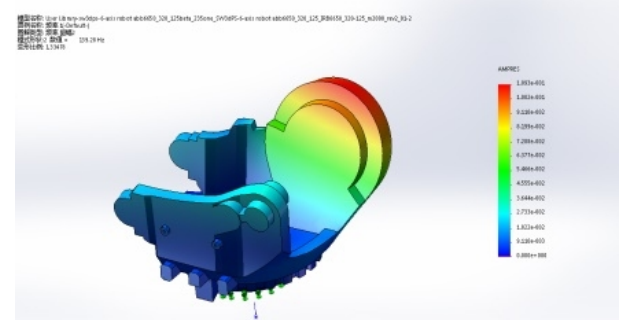

Fig.12 Distribution of vibration

\section{Strength check of typical parts}

Using the finite element analysis method to check the strength of important parts in Solidworks simulation.

1. Define material properties

2. Fixed constraint

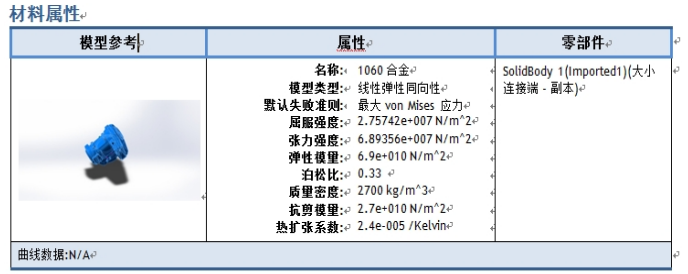

Fig.13 End Connection Material properties

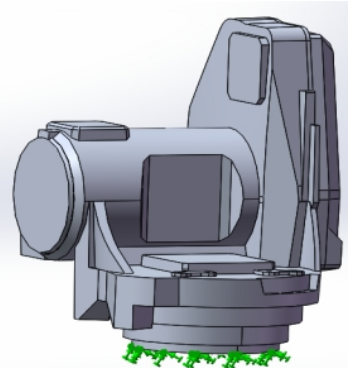

Fig.14 Constrained geometry

3. Calculated on Full-load, Exerting force 1000N

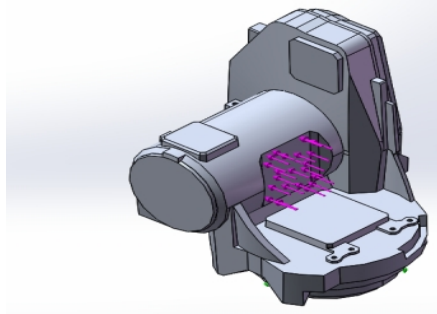

Fig.15 Applied load

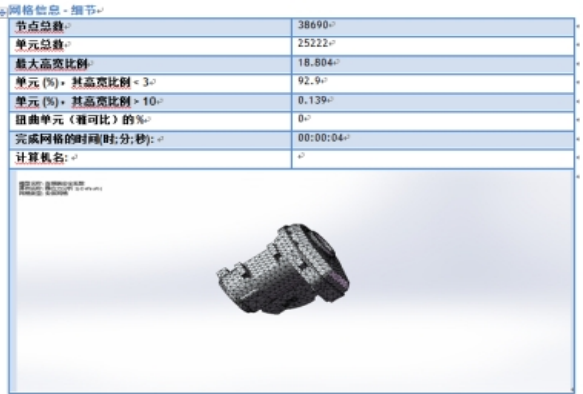

Fig.16 Mesh data

4. Dividing mesh

5. Calculated results 
The maximum stress appears in the ribs from the result, the numerical value is $4.17 \mathrm{e}+004 \mathrm{~N} / \mathrm{m}^{\wedge} 2$, and 1060 alloys allowable stress is $110 \mathrm{e}+006 \mathrm{~N} / \mathrm{m}^{\wedge}$ 2, within the allowable range.

6. Safety factor distribution

The minimum safety factor is about 66 from the analysis result as shown in Fig. 18 which is enough safe.

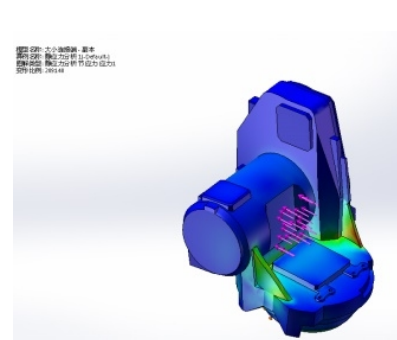

Fig.17 Calculation results of stress at arm end

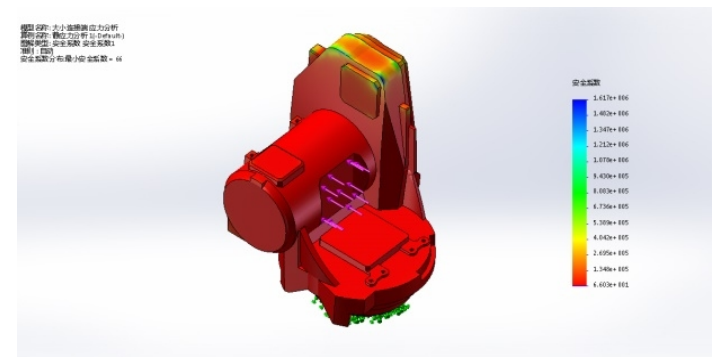

Fig.18 Safety factor distribution of arm end

By using the same method, Clamping manipulator finger material is selected, and the bending test is carried out. The analysis results are as follows.

According to the actual working conditions, the clamping mechanism is the biggest clamping pressure in the $100 \mathrm{~N}$. After applying the load, the calculation results are as Fig.20.

The safety factor distribution is shown in Fig.21, that shows the minimum safety factor is about 15, its strength is reliable, and it meets the requirements.

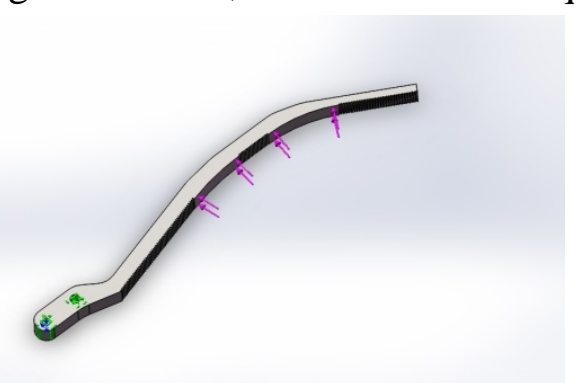

Fig.19 Finger load distribution

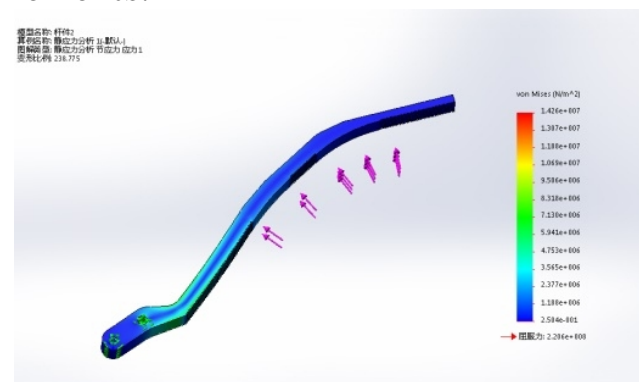

Fig.20 Calculation results of finger stress

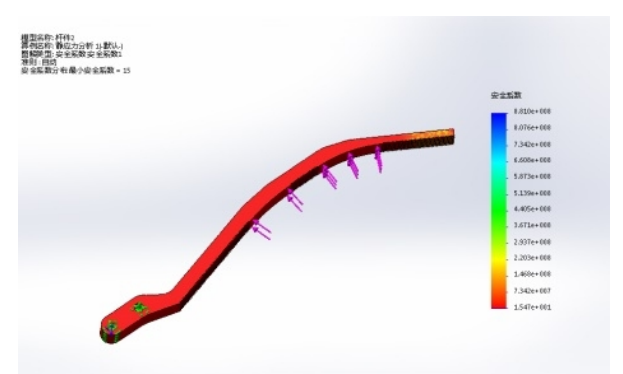

Fig.21 Safety factor distribution

\section{Mechanical arm movement simulation analysis}

\section{Robot arm motion simulation}

Robot arm movement simulation is the movement process of the mechanical arm which is simulated in SolidWorks software, which can observe the movement of the manipulator and the working situation in the virtual scene. The simulation movement analysis function in SolidWorks can help the user to design and assemble mechanism and carry on the simulation, including animation production, simulation of collision and interference and other physical properties of the object. 


\section{Simulation of the motion of grab workpiece}

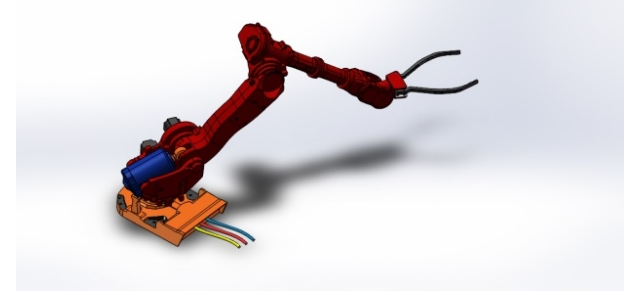

Fig.22 Motion simulation diagram

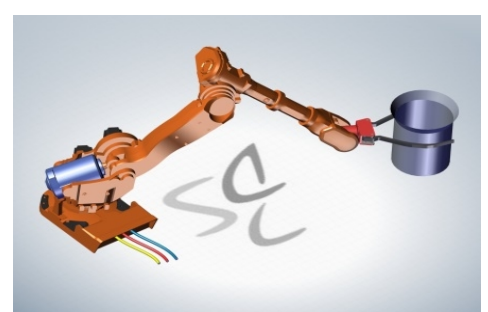

Fig.23 Working simulation diagram

\section{Simulated working scene}

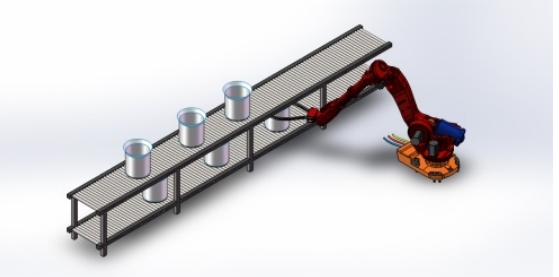

Fig.24 Simulated working scene

\section{conclusion}

In this paper, SolidWorks software is used to analyze the existing industrial robots, to find the breakthrough point and to expand the existing industrial robots.

1.Design and selection of workload and power source for mechanical arm: According to the given parameters such as the size and quality of the stamping part and the weight of the arm, then the parameters of the drive part can be selected. And select the appropriate servo motor and hydraulic cylinder.

2.Using Solidworks to design the shape and size of the components: The structure can be designed after the load and the motor are determined. Using SolidWorks software to draw the three-dimensional model of each part and determine the dimensions and other parameters, then to assemble each parts, finish assembly drawings, complete the design of the shape.

3.Using simulation SolidWorks to check the strength of important parts: To analyze the important parts in SolidWorks simulation when the structures design are completed, such as natural frequency analysis, bending test, torsion test, overturning test and so on.

4.The manipulator motion simulation: After the completion of the design steps, the manipulator is basically in line with the conditions of motion. The motion of the manipulator in the virtual scene can be accomplished by the motion of the SolidWorks.

\section{Acknowledgements}

This work was financially Supported by Jiangxi Province 2011 Collaborative Innovation Special Funds "Co Innovation Center of the South China Mountain Orchard Intelligent Management Technology and Equipment” ((Jiangxi Finance Refers to [2014] NO 156) ) ;

\section{References}

[1] confer editor, such as mechanical principle. Higher education press, 2001.

[2] Sun Jing democracy. Mechanical optimization design. The third edition. Beijing: mechanical indus try publishing house, 2005

[3] Solomon gathered. Industrial robots atlas. Beijing mechanical industry publishing house, 1993

[4] shih-chieh fang, yao-guang qi editor. Mechanical optimization design. Beijing: mechanical industr y press, 1997.2

[5] Wang Kun editor. Such as mechanical design course design manual. Beijing: mechanical industry publishing house, 2004 
[6] Cao Weiqing editor. Mechanism design. Machinery industry press, 2000.

[7] Juvinall r. c. Engineering Considerations of Stress, Strain and Strength. New York: McGraw Hill, 1967. 